\title{
Fake news: una revisión sistemática de la literatura
}

\section{Fake news: a systematic review of the literature}

\author{
Pablo Parra Valero*, Lídia Oliveira** \\ *Departamento de Biblioteconomía y Documentación, Universidad Complutense, España \\ **DigiMedia - Departamento de Comunicação e Arte, Universidade de Aveiro, Portugal
}

\begin{abstract}
The article is constructed from a systematic review of the literature on fake news and post-truth using the bases of scientific publications Web of Science and Scopus, accompanied by an analytical reflection. From the data collected in these two databases of publications shows the evolution of the number of publications on fake news and post-truth. Then, of the 482 documents of all typologies retrieved from Scopus, only the typology document in full text, totaling 91 , were considered. Based on their analysis, the ideas contained in them, the methodologies used and the proposals presented for fight the fake news. The results evidence that it was from 2016 that if its an exponential growth of publications double the topic of fake news and post truth. That the theme is treated transversally in several scientific areas, with more incidence in the area of communication, that a significant part of publications deal with case studies and also that a relevant part does not explain the methodology used. The solutions presented to overcome or mitigate the problem of fake news are of several natures: information literacy; algorithms and automatic procedures; crowdsourcing and professional verifiers.
\end{abstract}

Keywords: Fake news, post-truth, systematic literature review, Scopus, Web of Science

Resumen

El artículo se construye a partir de una revisión sistemática de la literatura sobre fake news y post-verdad usando las bases de publicaciones científicas Web of Science y Scopus, acompañada de una reflexión analítica. A partir de los datos recogidos en estas dos fuentes se muestra la evolución del número de publicaciones sobre fake news y post-verdad. Después, de los 482 documentos de todas las tipologías recuperadas de Scopus, sólo se consideraron los documentos de tipología artículo en texto completo, en un total de 91. A partir de su análisis se sistematizan las ideas en ellos contenidas, las metodologías utilizadas y las propuestas presentadas para combatir las fake news. Los resultados evidencian que fue a partir de 2016 cuando el crecimiento exponencial de publicaciones dobla el tema de la fake news y postverdad. El tema es tratado de forma transversal en diversas áreas científicas, con más incidencia en el área de la comunicación, además, una parte significativa de publicaciones abordan estudios de caso y otra no explicita la metodología utilizada. En cuanto a las soluciones presentadas para superar o mitigar el problema de las fake news son de diversa índole: alfabetización informacional; algoritmos y procedimientos automáticos; crowdsourcing y verificadores profesionales.

Palabras clave: Fake news, post-verdad, revisión sistemática de la literatura, Scopus, Web of Science

\section{Introducción}

Cada vez cuesta más discernir lo qué es verdadero de lo falso. El uso político, mediático y social de conceptos como posverdad o fake news es un problema global que amenaza a toda la población en su conjunto, a los medios de comunicación y a la propia Internet. Evidentemente, la existencia de noticias falsas no es nada nuevo, puesto que la difusión de la información sin contrastar es tan remota como nuestra existencia. De 
una manera u otra, es una realidad que siempre ha estado presente desde la Antigua Grecia (De los Santos, Smith, \& Cohen, 2018).

Pero, tal y como se conciben actualmente, las noticias falsas comenzaron a surgir en el siglo XIX en un momento de rápido crecimiento para los periódicos ayudado por las tecnologías emergentes en aquel entonces (Berkowitz \& Schwartz, 2016). Un ejemplo histórico fue la publicación en 1835 de una serie de seis entregas en el New York Sun que alertaban sobre la existencia de vida en la Luna (Allcott \& Gentzkow, 2017). Otro hito fue la famosa dramatización sobre la novela "La Guerra de los Mundos" de H.G. Wells, que un joven Orson Welles realizó en el estudio de la emisora CBS junto a otros compañeros la noche del 30 de octubre de 1938, en la víspera de Halloween. La lectura hizo sembrar el pánico durante varios minutos a millones de radioyentes estadounidenses que creyeron que una lluvia de meteoritos que contenían en su interior naves alienígenas estaba llegando a las calles de Nueva York y Nueva Jersey para someter el planeta con rayos y gases venenosos.

Pocos años más tarde, la publicación en la prensa de Filadelfia de informaciones falsas sobre ciudadanos irlandeses que robaban biblias en escuelas públicas norteamericanas ocasionaron varios disturbios (Bharali \& Goswami, 2018). Pero los considerados como pioneros en el arte de fabricar fake news fueron William Hearst y Joseph Pulitzer, el creador hace más de un siglo de los premios periodísticos que llevan su nombre. Ambos utilizaron sus respectivos periódicos el New York Herald y el New York World para difundir versiones sensacionalistas de los hechos y aumentar las ventas (Bhaskaran, Mishra, \& Nair, 2017).

Al analizar los principales periódicos durante las grandes confrontaciones del siglo XXI, se descubre con mediana claridad que las enormes mentiras y las grandes verdades han convivido con cierta naturalidad. Lo que se viene a denominar propaganda de guerra, que incluye también a la radio y a los pasquines que se lanzaban desde lo aviones, se fue transformando en otra manera de contar lo que sucedía en esos conflictos (Marcos Recio, Sánchez Vigil, \& Olivera Zaldua, 2017).

Desde finales del siglo XX conocemos la existencia de otro tipo de campañas de desinformación a gran escala relacionadas con el cambio climático, las vacunas, los alimentos, la nutrición, el origen de la vida, la salud, las armas en manos de los ciudadanos, los medicamentos genéricos, la curación u origen de enfermedades, la energía nuclear o el impacto de la inmigración. Pero sin duda, el momento más álgido del fenómeno de las fake news se vivió a partir de dos acontecimientos que sucedieron en 2016: el proceso del Brexit en Reino Unido y las elecciones norteamericanas que ganó Donald Trump. Uno de las numerosas investigaciones realizadas a partir de estas últimas, confirmaba que durante la campaña electoral se generaron un total de 115 noticias falsas favorables al actual presidente de Estados Unidos que se compartieron en Facebook un total de 30 millones de veces, frente a las 41 fake news en beneficio de Hillary Clinton compartidas en 7,6 millones de ocasiones (Allcott \& Gentzkow, 2017)

Por tanto, no es de extrañar la elección de posverdad (post-truth) y noticias falsas (fake news) como palabras del año en 2016 y 2017 por parte del prestigioso Oxford English Dictionary. El término post-truth fue usado por primera vez en un artículo del dramaturgo Steve Tesich sobre la primera guerra del Golfo Pérsico, publicado en 1992 en The Nation, la revista semanal más antigua de Estados Unidos (Berthon \& Pitt, 2018). En la definición del Oxford English Dictionary se hace referencia a "circunstancias en las que hechos objetivos influyen menos en la formación de la opinión pública que lo que lo hacen los llamamientos a emociones y creencias personales". Los responsables del prestigioso diccionario argumentaron que durante 2016 su uso aumentó un 2000\% con respecto a 2015. 
En 2017 los editores del Oxford English Dictionary seleccionaron el neologismo fake news como palabra del año, tras el aumento de su uso un 365\% en los últimos 12 meses, aunque un año antes, ya lo hizo en Australia el Macquarie Dictionary (Bolton \& Yaxley, 2017).

Otro aspecto más empírico que denota la importancia y el creciente interés por conceptos como posverdad o noticias falsas es el aumento exponencial de la producción científica sobre el tema a raíz del mencionado año 2016. Una búsqueda realizada por artículos de revistas sobre ambos conceptos en la plataforma Web of Science (WOS) el 9 de agosto de 2018 refleja la evolución del fenómeno.

Figura 1: Resultados recuperados de la búsqueda del término "post-truth" por tema en Web of Science y distribuidos por año de publicación

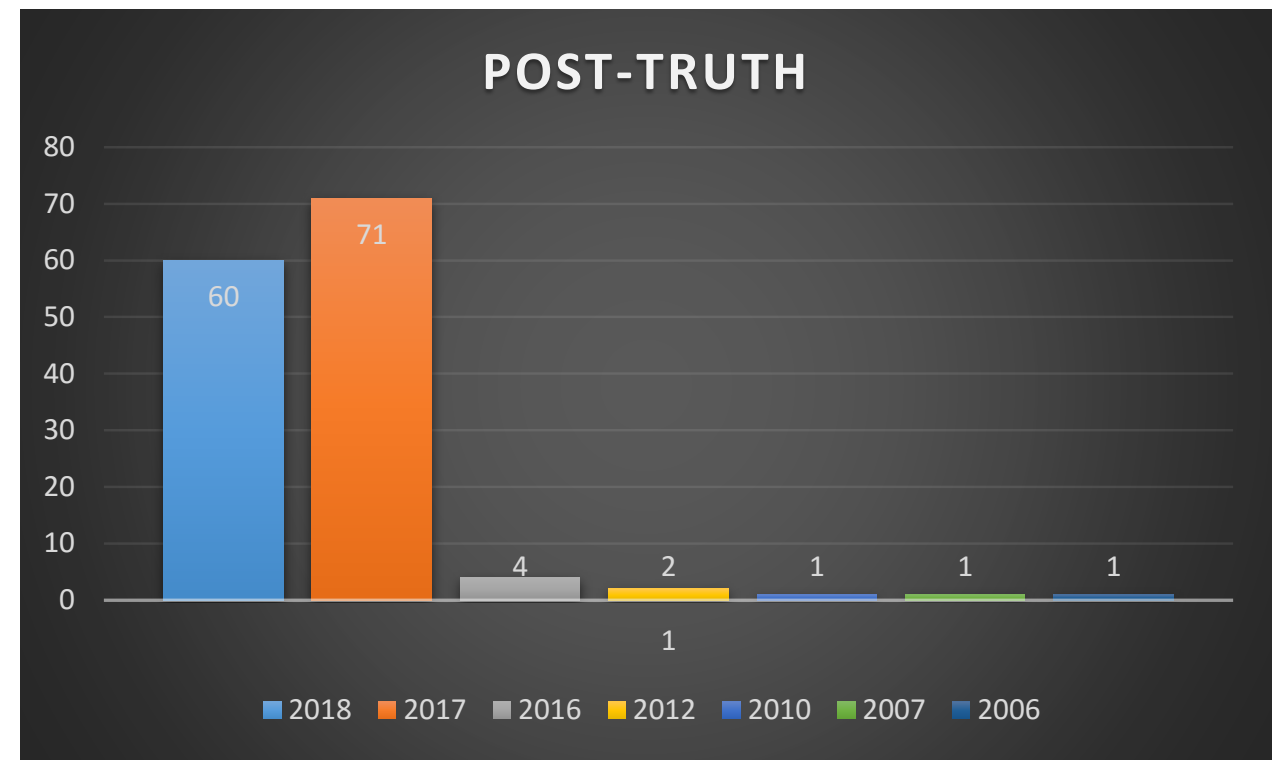

El número de artículos de revistas sobre fake news (150) era superior al de posverdad (140) y como se observa en la figura inferior, la tendencia apunta a que a finales de 2018 el número de artículos sobre noticias falsas duplicará la cifra de 2017. 
Figura 2: Resultados recuperados de la búsqueda del término "fake news" por tema en Web of Science y distribuidos por año de publicación

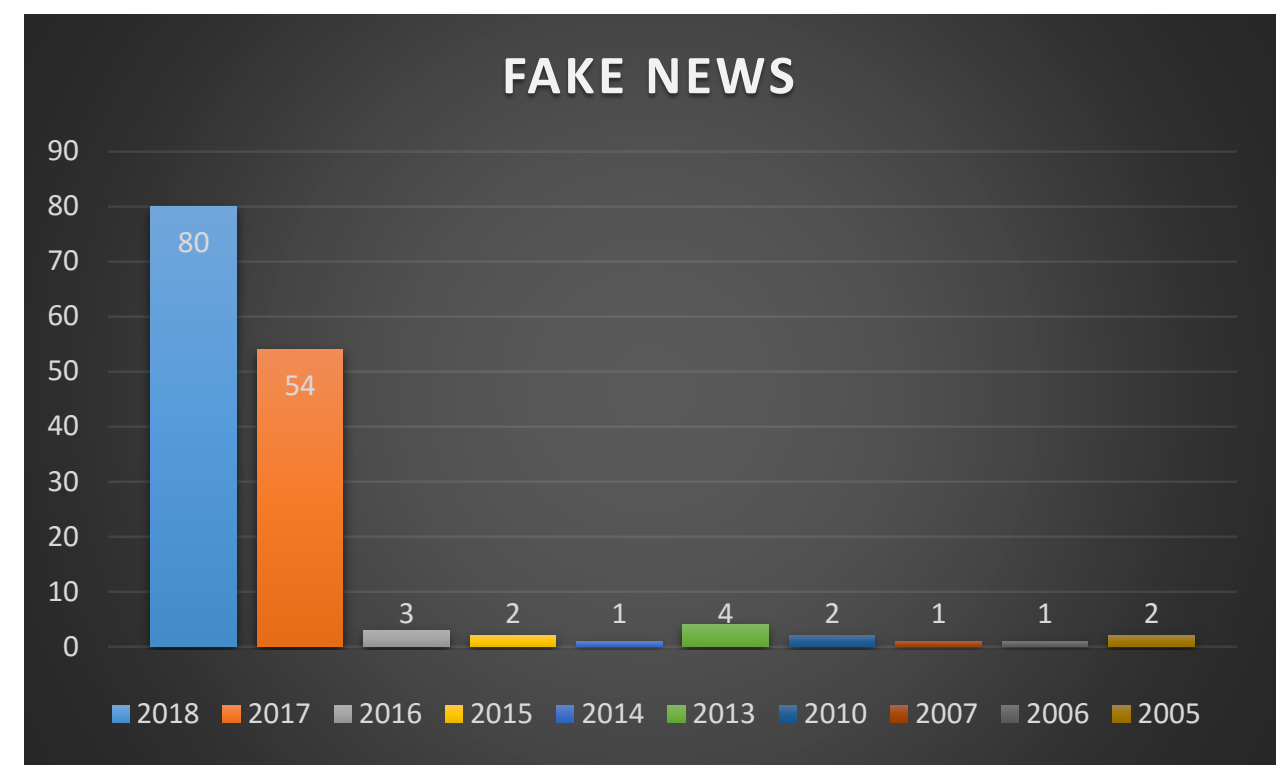

Al realizar el mismo proceso en la base de datos Scopus los resultados fueron muy parecidos. El número de artículos de revista localizados fue de 177 frente a los 129 encontrados en Web of Science.

Figura 3: Resultados recuperados de la búsqueda del término "post-truth" por título, resumen o palabra clave en Scopus y distribuidos por año de publicación

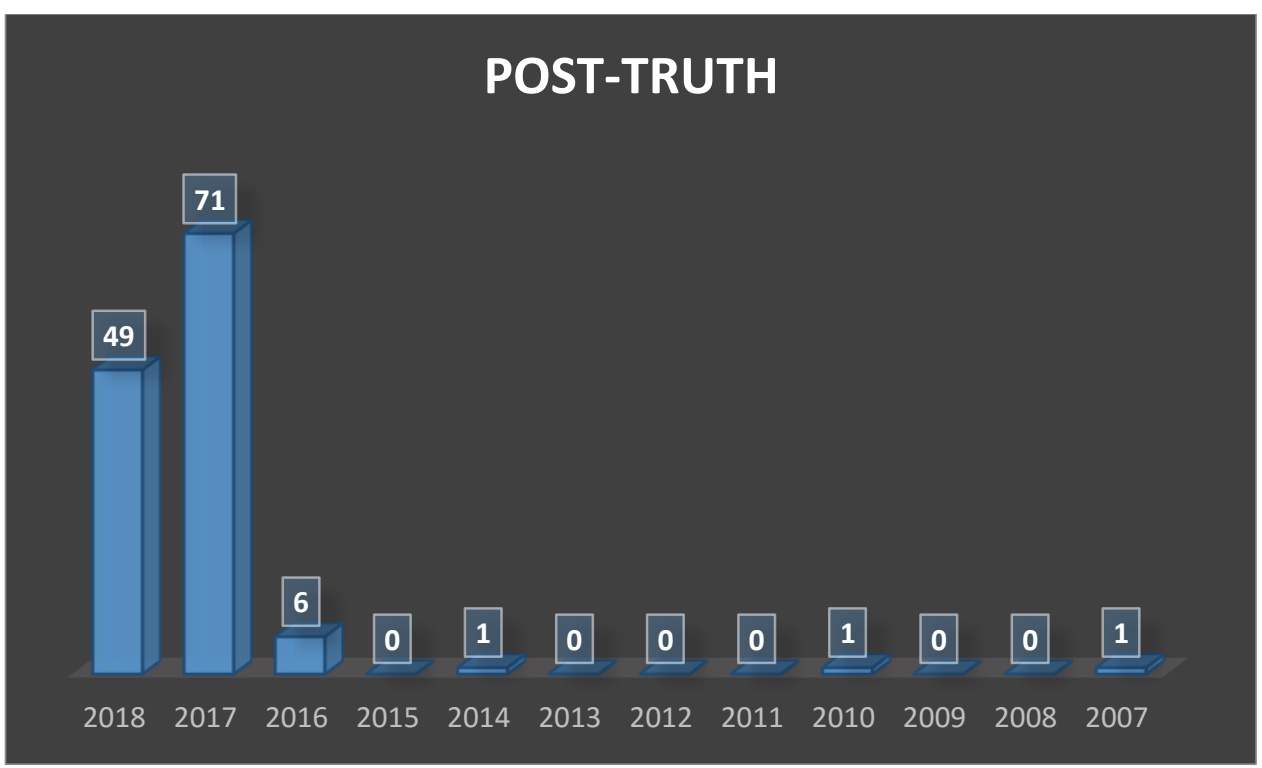


Los datos obtenidos tanto en Scopus como en Web of Science muestran como la presencia de trabajos científicos dedicados a noticas falsas ha sido más constante en la última década hasta llegar a 2018, año en el que la producción se ha elevado sobremanera.

Figura 4: Resultados recuperados de la búsqueda del término "fake news" por título, resumen o palabra clave en Scopus y distribuidos por año de publicación

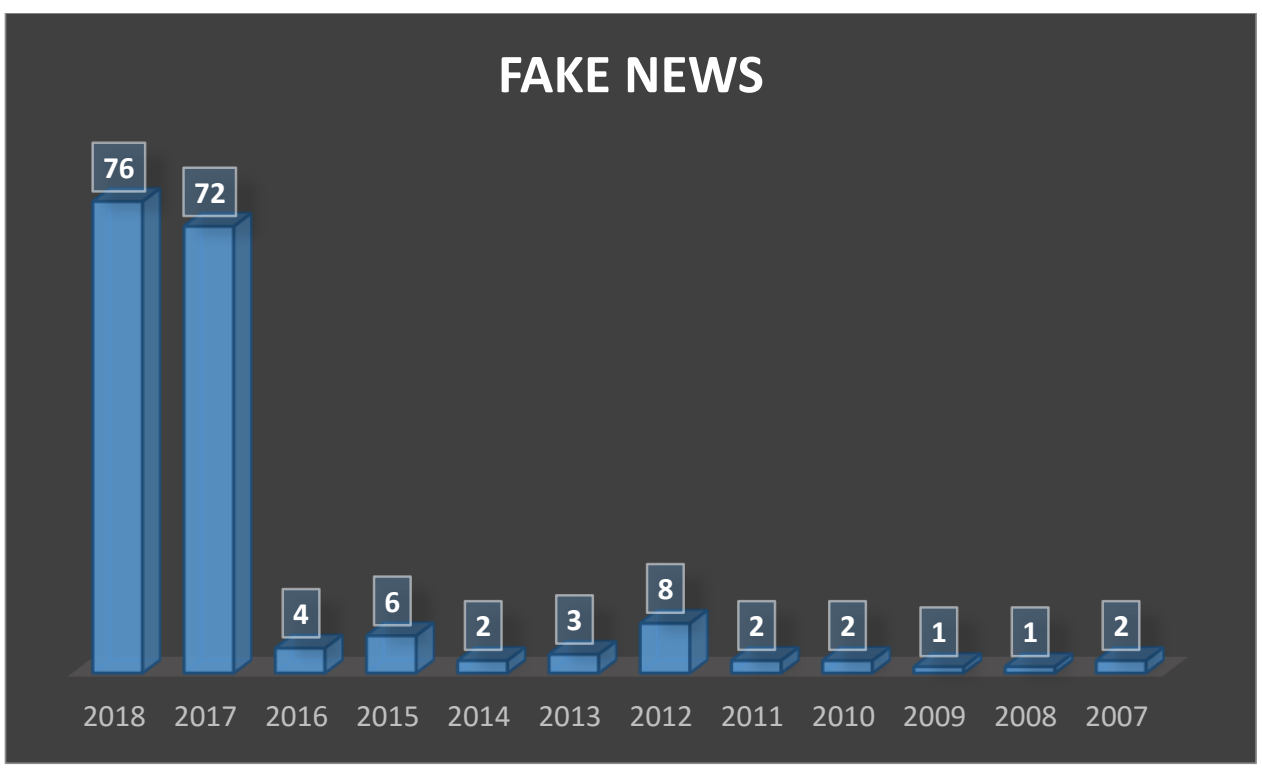

La permanente proliferación de noticias falsas que se manifiestan tras los sucesos señalados en 2016 a través de las redes sociales ha provocado que autoridades políticas de distintos países reclamen por la necesaria regulación del tema. Una primera reacción inmediata fue intentar censurar las noticias falsas y varios países como Irán, China, Italia o Alemania propusieron medidas legislativas al respecto (Andersdotter, 2017). Entre los gobiernos nacionales que se han decantado por la opción regulatoria destaca Alemania cuyo Gobierno presentó en marzo de 2017 un proyecto de ley que prevé multas de hasta 50 millones de euros a las redes sociales que no eliminen contenidos difamatorios, mensajes de odio o noticias falsas. En Italia se presentó una propuesta de ley para prevenir la manipulación de la información en línea y garantizar la transparencia en la web que incluye condenas civiles y penales para quienes difundan noticias exageradas o tendenciosas que incluyan datos o hechos manifiestamente infundados o falsos (Pauner Chulvi, 2018).

En Francia, el presidente Emmanuel Macron anunció el 3 de enero de 2018 la revisión de la legislación de los medios de comunicación franceses para combatir contra la difusión de noticias falsas en las redes sociales puesto que suponen una amenaza para las democracias liberales (Fischer, 2018). El pasado 4 de julio la nueva norma fue aprobada por la Asamblea nacional francesa. En concreto, una ley ordinaria para las campañas europeas y legislativas y otra, específica para las presidenciales. Según la nueva legislación, un candidato o partido podrá recurrir a un juez para que emita un recurso de urgencia ante la difusión de noticias falsas durante los tres meses anteriores a la elección del candidato o votación, siempre que pruebe que la difusión se realizó de manera deliberada. El texto ha sido criticado por la oposición en el parlamento 
francés, tanto de los partidos de izquierda como de la derecha, así como por los medios de información y los sindicatos que lo consideran un grave peligro para la libertad de expresión.

Conscientes del limitado impacto y eficacia que una lucha a nivel nacional contra las noticias falsas puede tener en la época de la globalización, el ámbito de actuación también se ha ampliado a la Unión Europea. Desde las instituciones comunitarias se ha lanzado una llamada de colaboración a las grandes empresas tecnológicas para frenar la propagación de informaciones fraudulentas que abundan en Internet y se ha reclamado un compromiso similar al seguido en la lucha contra la propagación de mensajes que fomentan el odio, el racismo y la xenofobia a través de las redes sociales. Tras analizar algunas propuestas generales (autorregulación, acciones legales y multas), la Comisión Europea decidió lanzar una consulta pública a un grupo de expertos para que emitieran un informe con recomendaciones para combatir las fake news. Para ello, en enero de 2018 se convocó a 39 periodistas, profesionales en la identificación de bulos, dirigentes de medios de comunicación y políticos. El resultado fue un informe titulado "Un enfoque multidisciplinar a la desinformación"1 que incluye una serie de recomendaciones entre las que destacan:

- El abandono del término fake news ya que no explica la complejidad del problema y genera confusión en los debates políticos, mediáticos y académicos. Es preferible hablar de desinformación.

- Apoyo financiero para iniciativas de medios independientes de verificación y programas educativos de alfabetización mediática y tecnológica.

- Un llamamiento para que las plataformas como Google, Facebook y Twitter proporcionen datos que permiten estudiar el impacto de la desinformación y sus posibles soluciones.

- Una petición para que las instituciones públicas a todos los niveles de la Unión Europea compartan los datos de manera rápida y eficiente cuando así lo soliciten las organizaciones de verificación de datos.

- Creación de una red de centros de investigación sobre desinformación en la Unión Europea.

- Rechazo a cualquier intento de censura de contenidos

En el ámbito internacional, la ONU en el año 2017 también expresó su preocupación por el tema en una "Declaración conjunta sobre Libertad de Expresión y Noticias Falsas, Desinformación y Propaganda" (Pauner Chulvi, 2018).

En consecuencia, la proliferación de noticias falsas ha establecido un cambio cultural significativo en el ámbito de la política y la información (Cosentino, 2012). Las redes sociales han modificado radicalmente el paradigma del consumo de noticias (Cardoso et al., 2018). Según un reciente estudio del Instituto de

\footnotetext{
${ }^{1}$ Disponible en:https://www.ami.info/wpcontent/uploads/2018/03/HLEGReportonFakeNewsandOnlineDisinformation.pdf (accedido en 2018-08-23).
} 
Tecnología de Massachusetts la difusión de fake news puede ser retuiteadas o compartidas en redes sociales hasta un $70 \%$ más que las verdaderas. Además, desde hace una década, se ha producido un cambio extraordinario en el diseño de negocio de la prensa que no rentabiliza su tradicional patrón publicitario y se enfrenta a nuevas exigencias del público, distribución y comercialización. Mientras las grandes empresas de comunicación están respondiendo a una caída generalizada de anunciantes y suscriptores con recortes de personal o reconfiguraciones de plantilla más o menos drásticas. Esta transformación también ha alcanzado a los nuevos modos de producción narrativa de la prensa basada ahora en la rapidez o casi instantaneidad informativa. Con el advenimiento del entorno digital y la necesidad de una información permanentemente actualizada se ha quebrado la precisión que requiere la elaboración de las noticias, una de las condiciones básicas para ofrecer información de calidad y sin errores (Pauner Chulvi, 2018).

\section{Metodologia}

Dada la cantidad de afectados por el problema de las fake news y la amenaza que supone para la sociedad, el objetivo general de esta investigación es analizar las soluciones que se plantean a través de un estudio de la producción científica surgida fundamentalmente en los dos últimos años. Como punto de partida para hacer esta revisión bibliográfica se consultan las bases de datos Web of Science y Scopus, puesto que constituyen las dos principales fuentes de información internacionales a nivel académico. En la figura inferior generada desde Web of Science a partir de los artículos de revistas obtenidos sobre fake news se aprecia el carácter multidisciplinar del tema.

Figura 5: Resultados recuperados por la búsqueda del término "fake news" distribuidos por áreas de conocimiento en Web of Science

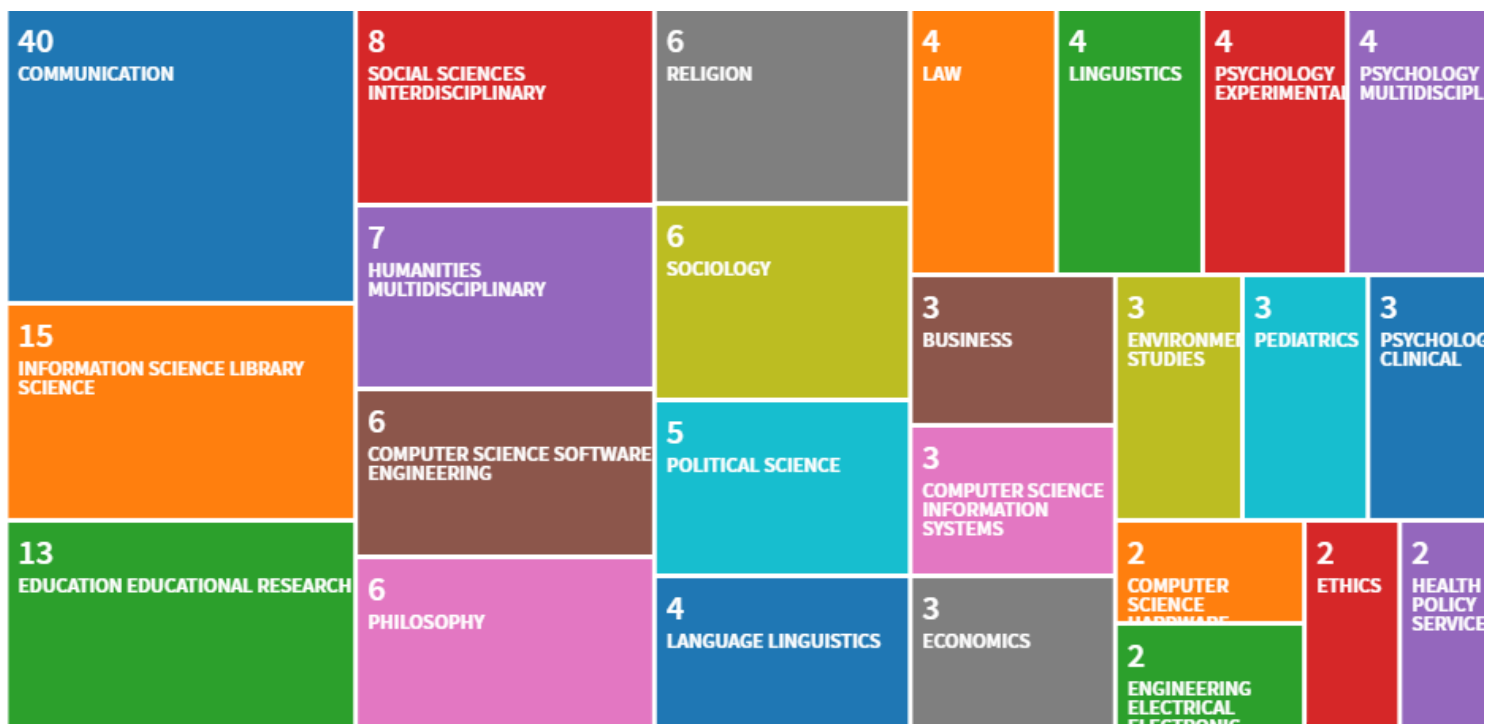


Siendo Scopus hoy la mayor base de datos multidisciplinar y por poseer una mayor cobertura por número de revistas y citas por artículo que Web of Science (Hernández-González et al., 2016) se eligió esta fuente de información. De esta manera, el 9 de agosto de 2018 se realizó en Scopus una búsqueda bibliográfica para localizar todos los artículos de revistas que contuvieran el término fake news en el título, en el resumen o en las palabras clave. Con una representatividad del $36,7 \%$, el análisis se centra únicamente en esta tipología.

Figura 6: Resultados obtenidos en Scopus por tipología documental

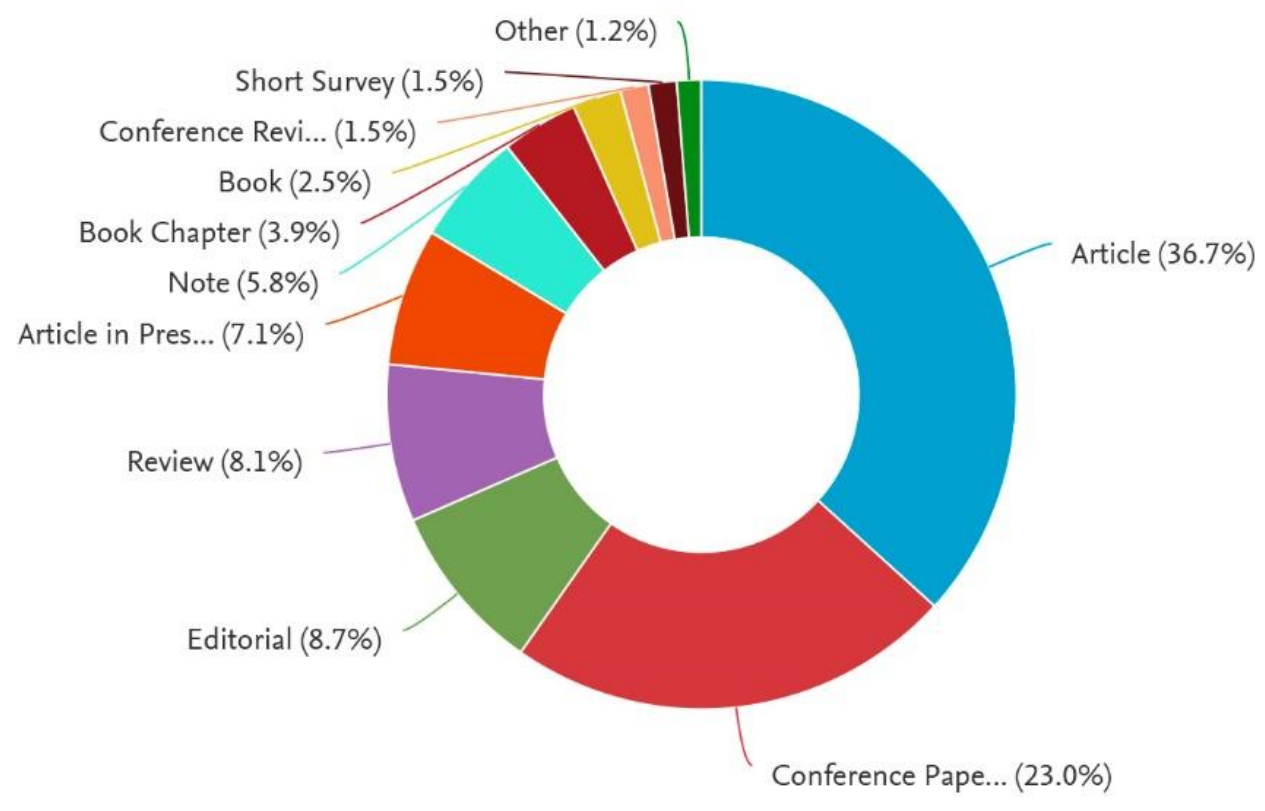

No obstante, la revisión previa del resto de fuentes de información que recoge Scopus considerados como la presencia de un 7,1 \% de documentos que abordaban cuestiones relacionadas con el tema de la medicina y la salud, si bien es cierto que muchos autores tienden a emplear el termino fake news simplemente como un mero efecto de llamada o atención en el título o en el resumen. 
Figura 7: Artículos de revistas sobre "fake news" en Scopus distribuidos por áreas de conocimiento

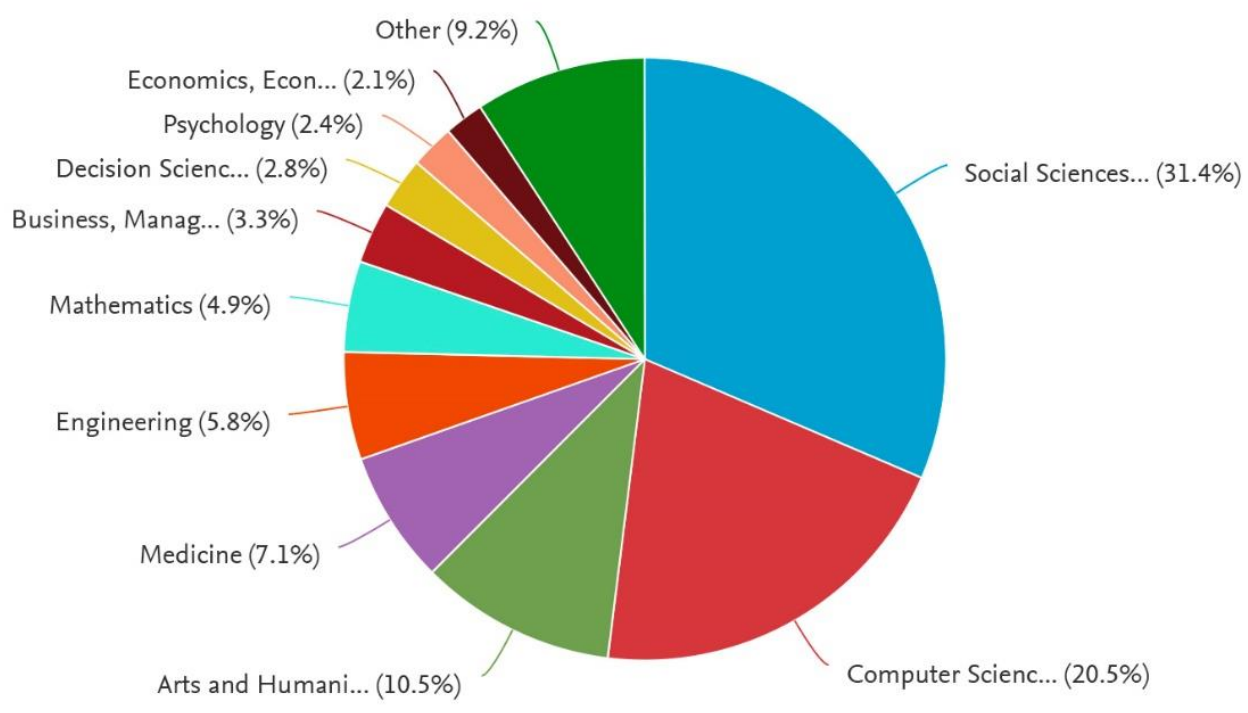

También se ha constatado como diversas divulgaciones recientes alimentan la afirmación de que los hechos científicos están bajo sospecha y en crisis. Para algunos autores, los científicos parecen involucrarse tanto en interés fácticos como en revelar hechos interesantes y vemos como investigadores publican continuamente resultados positivos, mientras que otros publican resultados negativos en el mismo problema, incluso basándose en los mismos datos (Hofmann, 2018).

Esta lectura y revisión previa de los 482 documentos de las distintas tipologías documentales encontradas en Scopus permitió además categorizar una serie de aspectos para elaborar nuestro estudio y poder responder a las siguientes cuestiones:

- ¿Cuántos artículos están basados en experimentos ejecutados en la práctica?

- ¿Qué número de artículos corresponde a análisis o estudios de casos?

- ¿Cuántos artículos detallan la metodología empleada?

- ¿Cuántos artículos abordan la definición de fake news?

- ¿Los artículos plantean como resolver el problema de las noticias falsas?

- ¿Cuáles son los ejemplos de noticias falsas que más se citan?

Finalmente, de los 178 artículos de revistas obtenidos a través de Scopus, se aplicó un análisis de contenido a un total de 91 , todos los disponibles a texto completo.

\section{Resultados}

Una de las observaciones más llamativas es la frecuente alusión al presidente de los Estados Unidos o al proceso que tuvo lugar en 2016 y que han otorgado una nueva dimensión al tema de las noticias falsas. Un 
total de 40 de los 91 documentos analizados contenían el nombre del actual presidente de los Estados Unidos.

Figura 8: Cuantidad de artículos que aluden a las últimas elecciones presidenciales de Estados Unidos

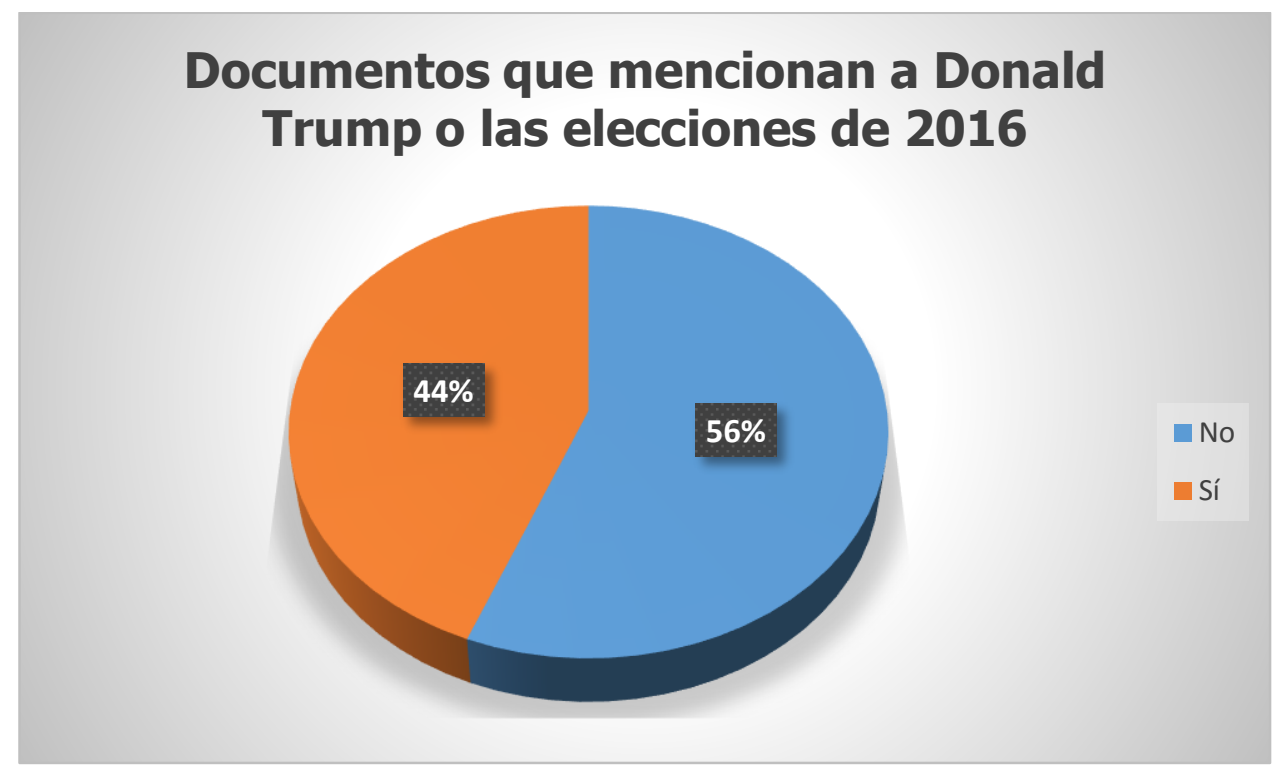

Desde entonces, comprobamos que los procesos electorales vividos en otros países se han visto enturbiados por campañas de desinformación como así ha sucedido en Alemania, Austria, Hungría, México, el plebiscito por la paz en Colombia (Pauner Chulvi, 2018), la propuesta de reforma constitucional de Italia (Del Vicario et al., 2018), el referéndum de Cataluña o el "Penelopegate" o caso Fillon en 2017 en Francia (Baider \& Constantinou, 2017), entre otros.

Al margen de la dimensión política, la revisión de la bibliografía científica que ofrece Scopus ha permitido recopilar un buen número de otros casos de noticias falsas, entre los que destacan: la afirmación de que las vacunas causan autismo (Arif et al., 2018) u otros efectos perniciosos (Krishna, 2017) (Bozzola et al., 2018); la participación del gobierno de Estados Unidos en los ataques terroristas del 11 de septiembre de 2001 (De los Santos et al., 2018); los atentados de Boston en 2003 (Himma-Kadakas, 2017) (Vosoughi, Mohsenvand, \& Roy, 2017) (Pantumsinchai, 2018); la publicación de la fotografía del cadaver de Osama Bin Laden (Brandtzaeg, Følstad, \& Chaparro Domínguez, 2017); el atentado que tuvo lugar el 17 de agosto de 2015 en el centro de Bangkok (Pantumsinchai, 2018); la epidemia del Ébola en 2014 (Vosoughi, Mohsenvand, \& Roy, 2017)(Waldrop, 2017); el virus del Zika en 2015 y 2016 (Sommariva et al., 2018); el uso de opiáceos durante el embarazo (Arndt \& Jones, 2018); el daño potencial del veneno de las arañas (Hauke \& Herzig, 2017); los desórdenes en la localidad estadounidense de Ferguson el 9 de agosto de 2014 tras el asesinato del joven afroamericano Michael Brown, (Vosoughi, Mohsenvand, \& Roy, 2017); el tiroteo en la Escuela Primaria Sandy Hook de Newton (Connecticut) que acabó con 26 personas el 14 de diciembre de 2012 (Himma-Kadakas, 2017); los disturbios en Reino Unido iniciados el 6 de agosto de 2011 a raíz del fallecimiento de Mark Duggan, un joven de 29 años de raza negra que murió por disparos de la Policía Metropolitana de Londres (Himma-Kadakas, 2017); la crisis en Ucrania de 2013-2015 (Himma-Kadakas, 
2017)(McNair, 2017) o el temor a los refugiados y a los solicitantes de asilo en los países europeos (McBrien, Dooley, \& Birman, 2017).

En menor medida, también conviene señalar como un $8 \%$ de los artículos de revista están dedicados a analizar los programas televisivos o medios de comunicación que se encargan de propagar este tipo de noticias de una manera cómica. La comedia tiene un papel esencial en ayudar a las sociedades a gestionar los momentos de crisis y en Estados Unidos después del 11-S muchos medios satíricos contribuyeron a superar la tragedia (Achter, 2008). Uno de ellos fue The Onion. Surgido como un diario de entretenimiento, actualmente es una web dedicada a la creación de bromas que todo lo que publica es falso y que incluso contrata a presentadores de programas informativos para leer sus fake news (Berkowitz \& Schwartz, 2016). Junto a este espacio, encontramos late nights con más tradición como el Saturday Night Live que se ha convertido en uno de los programas de noticias falsos más icónicos (Day \& Thompson, 2012) o The Daily Show el más citado en los artículos analizados y el más popular de la televisión norteamericana. Se trata de un programa de noticias falso que usa la sátira para interrogar al poder, la parodia para criticar noticias contemporáneas y el diálogo para promulgar un modelo de democracia deliberativa (Baym, 2005). El éxito del formato de estos espacios ha permitido su reproducción en otros países con distinto éxito como es el caso del programa británico Brass Eye (Meikle, 2012), los australianos The Norman Gunston Show y Newstopia (Harrington, 2012), el italiano Striscia la Notizia, uno de los pioneros en el género (Cosentino, 2012) y su versión rumana Cronica Cârcotaşilor (Bardan, 2012).

De los 91 artículos de revista a texto completo analizados sobre fake news accesibles a través de Scopus, más de un tercio (37\%) corresponde a estudios o análisis de casos. En cambio, tan solo se dieron 4 casos $(4,3 \%)$ realizados a partir de experimentos reales:

- Experimento con 60 participantes, divididos en partes iguales entre médicos, enfermeras y estudiantes de medicina, cada uno de los cuales estuvo expuesto a ocho rumores relacionados con el cáncer (Chua \& Banerjee, 2018)

- Experimento con 400 participantes para identificar como las personas son más vulnerables a los efectos persistentes de las informaciones falsas.

- Experimento con 40 estudiantes de psicología que fueron expuestos a una serie de noticias falsas (Polage, 2012).

- Experimento basado en crowdsourcing a través de la plataforma Mechanical Turk de Amazon a partir de diez hallazgos científicos vinculados con fake news (Verma, Fleischmann, \& Koltai, 2017).

De todos los artículos trabajos basados en experimentos o estudios de caso tan solo un $21 \%$ detallan la metodología empleada. 
Figura 9: Artículos de revistas en Scopus que describen la metodología empleada

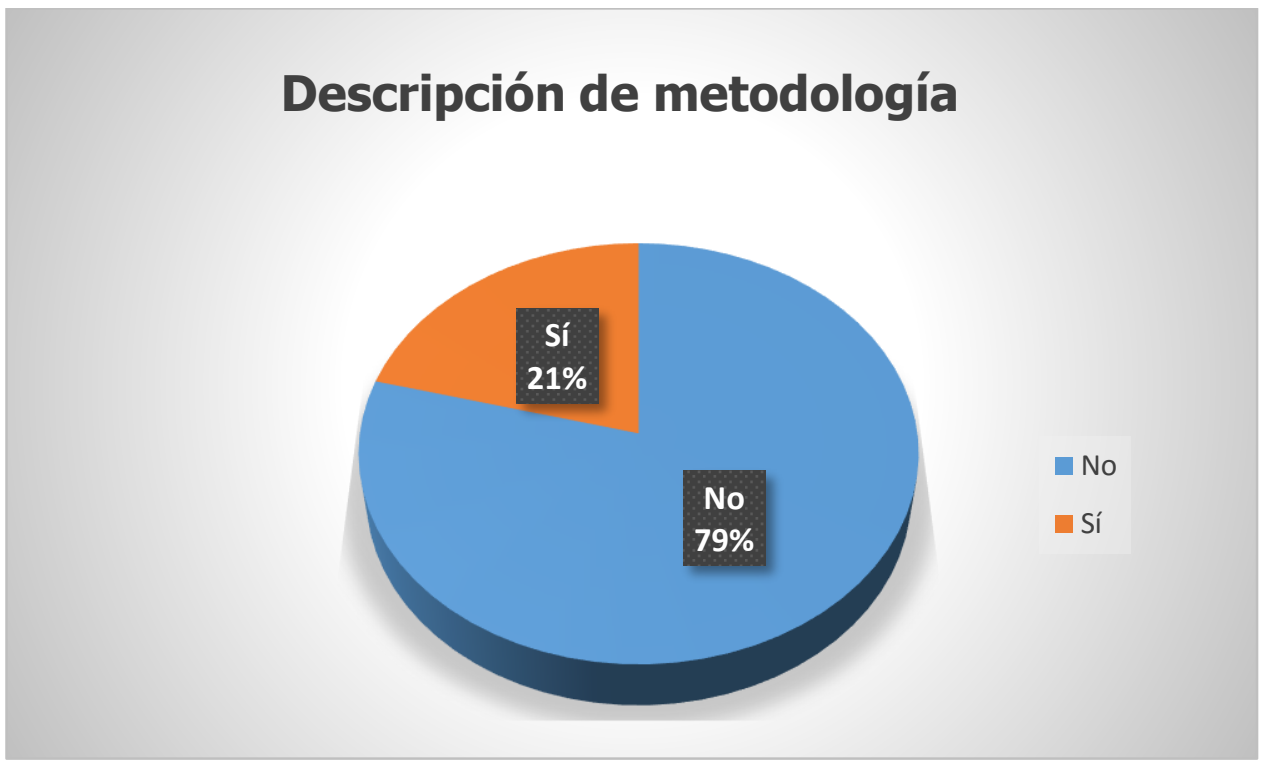

En cuanto a la terminología, pese a que existe mucha confusión sorprende el escaso número de artículos (8\%) que recogen su definición. Las noticias falsas no son solo prejuicios, sátiras o parodias. Algunos autores defienden que simplemente es una nueva denominación del periodismo amarillo acuñado en la década de 1890, aunque este tipo de información ya existía desde mucho antes (Ireland, 2018). Para otros los neologismos fake news y posverdad tienen una importante variedad de definiciones y el primero ha adquirido significados múltiples y muy politizados desde las últimas elecciones presidenciales norteamericanas (Johnson \& Kelling, 2017).

Sin embargo, ambos mantienen una relación cercana y complementaria ya que, aunque son términos que aluden a realidades y sujetos distintos, muy habitualmente se da entre ellos una correlación temporal. El objetivo de las noticias falsas es influir en la opinión pública y crear inseguridad, sospechas, desestabilización para apoyar o desacreditar o, a veces, simplemente diversión. En última instancia, son un medio para obtener seguidores, perfiles y usuarios de redes sociales para alentar un movimiento, un interés económico o de marca o a una persona. La posverdad es un elemento disruptivo y perturbador que produce un efecto desestabilizador y una respuesta irracional en la sociedad. En este sentido, el término ha venido reflejando que aquello que las personas sienten ante un estímulo, sus emociones y creencias a respecto de una idea o de un líder, o sus sensaciones subjetivas influyen de una forma más efectiva en la toma de sus decisiones y son más importantes que la verdad (Pauner Chulvi, 2018). El prefijo "post" ha sido reconocido en el discurso social, político y académico desde mediados del siglo pasado, por ejemplo, en términos como postnational o post-racial cuyo significado alude a un tiempo en el que los conceptos especificados son irrelevantes o han perdido importancia. Según Neil Midgley, la palabra posverdad posee el mismo matiz, tras unas décadas de silencio en los discursos políticos ha explotado en la conciencia dominante a raíz de las consecuencias del referéndum del Brexit y las elecciones presidenciales de Estados Unidos (Verrall \& Mason, 2018). 
Por otro lado, redes sociales como Facebook, YouTube, Instagram y Twitter cada vez se utilizan más como fuentes de información tanto por los medios de comunicación como por las personas en general. A raíz de los hechos acaecidos tras las elecciones norteamericanas, el porcentaje de usuarios que usaron las redes sociales en 2016 casi duplica los registros del año 2013 (Brandtzaeg, Følstad, \& Chaparro Domínguez, 2017). Entre los numerosos ejemplos de datos estadísticos ofrecidos en los diferentes artículos hallados sobre la gravedad del tema, destacamos el realizado por el Pew Research Center que registró que el $62 \%$ de los adultos estadounidenses recibe sus noticias a través de las redes sociales o que el $66 \%$ de los usuarios de Facebook usan la plataforma para el consumo de noticias (Spohr, 2017). Otro estudio reciente confirma que las noticias falsas más populares fueron más compartidas en Facebook que la noticias reales más populares (Allcott \& Gentzkow, 2017). En España según la Asociación para la Investigación de Medios de Comunicación (AIMC), el $56,6 \%$ de los internautas utiliza las redes para informarse y para un $70 \%$ Internet es ya su fuente principal de información.

Buena parte del problema es el hecho de que empresas como Google o Facebook se han apropiado y monopolizan el mercado de la publicidad en la red. Esto ha originado un modelo de negocio de pago por uso, en el que los anunciantes solo cobran cuando una página es vista o se hace click en ella (clickbait), por lo que muchas empresas de comunicación cuentan con pocos incentivos para realizar su tradicional función de observadores de la realidad. Hace unos meses el vicepresidente de Google News, Richard Gingras afirmaba que el mayor problema sobre las noticias falsas y la desinformación es que se distribuyen en un ambiente político en el que, en muchas ocasiones, amenazan la libertad de expresión. Con el propósito de solucionar los problemas que en los últimos meses han afectado a la compañía en relación con las fake new, la plataforma de videos YouTube anunció el pasado 10 de julio que invertirá 20 millones de euros para luchar contra las noticias falsas. Entre las medidas anunciadas, destacan la incorporación de enlaces a los vídeos que irán acompañados de pequeños extractos de noticias fiables.

Ante todas estas disyuntivas cabe plantearse cuál es la manera de resolver el problema de las noticias falsas. En el presente estudio el porcentaje de artículos de revistas analizados a través de Scopus que proponen algún tipo de solución es del 34\%. Según Pauner Chulvi (2018) las propuestas concretas que están implementando las redes sociales en la lucha contra las noticias falsas gravitan en torno a tres opciones: uno, el reclutamiento de un grupo humano de revisores o editores especialmente procedentes de los medios de comunicación tradicionales; dos, una estrategia colaborativa y tres, soluciones tecnológicas basadas en algoritmos.

En relación a las soluciones humanas se trataría de la incorporación de equipos de verificadores o profesionales capacitados para comprobar y contrastar los datos e informaciones que un medio obtiene de forma abierta o confidencial, de fuentes oficiales o través de la web social. En este sentido, la revisión sistemática ha permitido constatar el actual funcionamiento de múltiples herramientas, proyectos y portales dedicados a combatir la desinformación, entre los que podemos destacar los siguientes:

- Snopes. Página de investigación sobre leyendas urbanas. Es una de las más populares a nivel mundial relacionadas con desarmar bulos, rumores y noticias falsas

- Stopfake. Sitio web que comprueba hechos periodísticos sobre la guerra entre Rusia y Ucrania.

- FactCheck. Sitio web sin fines lucrativos que tiene como objetivo reducir el nivel de engaño y confusión en la política estadounidense 
- Politifact. Sitio web propiedad del diario Tampa Bay Times de Florida ganador de un Premio Pulitzar en el año 2009. Se dedica a confirmar la veracidad de las afirmaciones de políticos y analistas, midiéndolas con su herramienta Truth-O-Meter

Tras estas variadas iniciativas hay diferentes personas, medios de comunicación o incluso instituciones educativas como la University of Pennnsylvania creadora de FactCheck en 2003, pero quizá la más conocida es Stopfake a cuyo proyecto se han unido periodistas, empresas, programadores, traductores y otras muchas personas preocupadas por el destino de Ucrania.

En cuanto a las soluciones colaborativas (crowdsourcing) se trata de abrir el proceso de evaluación de las noticias a los usuarios de las redes permitiéndoles calificarlas según su criterio y decidir si les otorga o no credibilidad. El etiquetado de las noticias es un proceso de construcción voluntaria similar al que sigue Wikipedia, abierto teóricamente a todo el mundo, pero también susceptible de ser manipulado por grupos de personas que promuevan las mismas noticias falsas (Pauner Chulvi, 2018).

Sobre las soluciones basadas en procedimientos automáticos y el desarrollo de algoritmos, si bien las compañías tecnológicas afirman que están libres de sesgo ideológico, es incuestionable que no son necesariamente equitativos puesto que quienes los crean definen su funcionamiento y sus resultados. Las ventajas de esta opción son su bajo coste económico y la facilidad de su implementación. Por otra parte, algunos estudios evidencian que los materiales informativos elaborados mediante inteligencia artificial resultan más creíbles que los elaborados por humanos (Túñez-López et al., 2018). Entre las herramientas de curación automática destaca Hoaxy, un motor de búsqueda desarrollado por la Indiana University que permite buscar y hacer un seguimiento de noticias falsas con el objetivo de analizar el problema y poder diseñar estrategias que acaben con la desinformación. El sistema emplea una serie de rastreadores web para detectar enlaces a artículos ubicados en sitios de noticias falsas y mostrar cómo éstas se vuelven virales. Además, Hoaxy proporciona dos tipos de visualización (una línea de tiempo que muestra el crecimiento del número de tweets de artículos de escasa credibilidad, y una visualización interactiva de la red de difusión, pero también conviene señalar que los artículos recopilados no se verifican (Shao et al., 2018).

Entre los estudios de casos revisados que plantean el empleo de algoritmos destacamos Rumor Gauge un sistema para la verificación automática en Twitter que fue probado en 209 ejemplos reales, prediciendo correctamente la veracidad del 75\% de los rumores (Vosoughi, Mohsenvand, \& Roy, 2017). En la búsqueda de un método de evaluación de alta precisión algunos autores abogan por un enfoque híbrido e innovador que combine enseñanza lingüística y modelos precisos de aprendizaje automático a partir de datos de comportamiento basados en metadatos y consultas en la red (Chen, Conroy, \& Rubin, 2015).

En nuestro análisis bibliométrico realizado a partir de los resultados encontrados en Scopus sobre fake news se observa como la opción de los algoritmos y la inteligencia artificial es una de los dos que gozan de mayor tratamiento. 
Figura 9: Posibles soluciones mencionadas en los artículos de revista

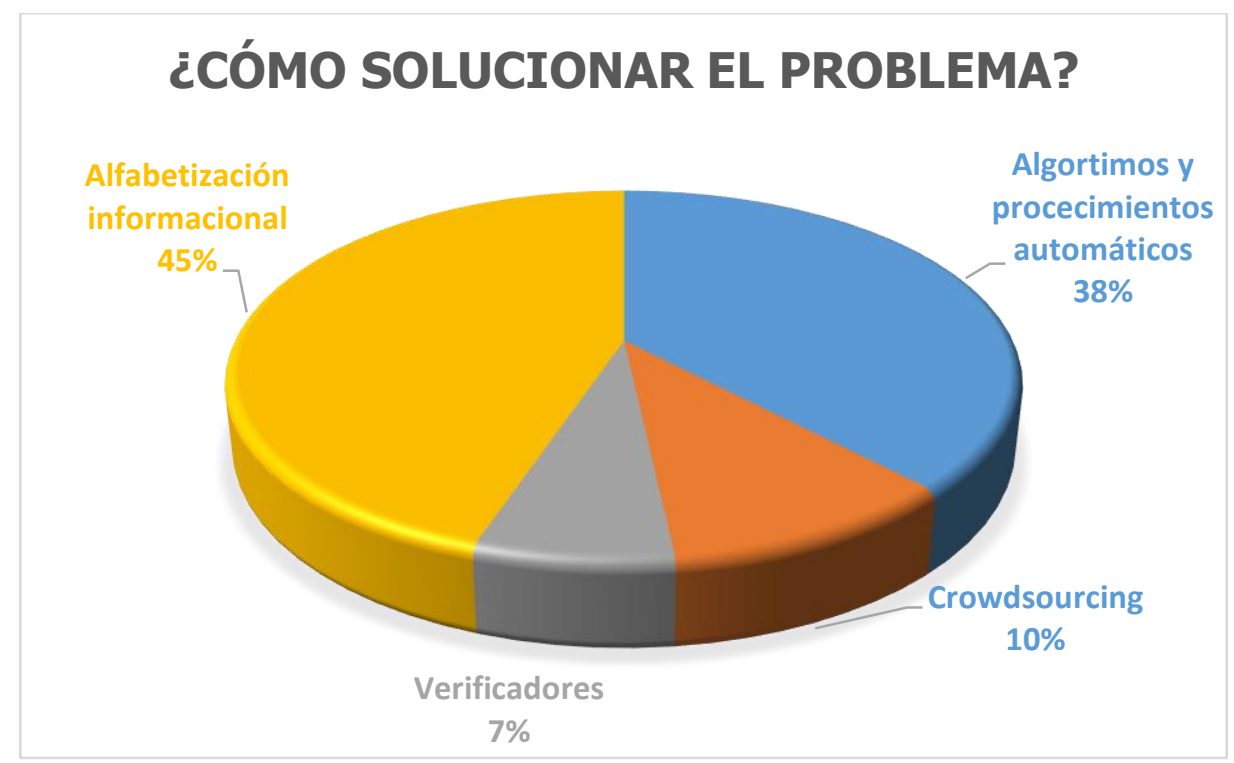

Por delante de la aplicación de algoritmos y con casi la mitad de resultados, la solución más repetida que se deriva de las publicaciones científicas analizadas para poder mitigar el problema al que nos enfrentamos, es el papel que pueden ejercer las competencias informacionales de los profesionales de la documentación. Especialmente, los bibliotecarios puesto que poseen la responsabilidad profesional de promover habilidades de pensamiento crítico entre la población. En el ámbito anglosajón el debate sobre las noticias falsas ha traído una nueva atención sobre la alfabetización mediática y el papel de las bibliotecas y otras instituciones. Desde su origen, los bibliotecarios siempre han enseñado y ayudado a los usuarios a encontrar y entender la información que necesitan. En este proceso actual que las nuevas tecnologías imponen a nuestra profesión, la respuesta profesional ha sido la de reafirmar los valores básicos y mantener los servicios tradicionales como una forma de combatir la desinformación. Ahora, surge un nuevo desafío en el que los bibliotecarios pueden desempeñar un papel fundamental en el desarrollo de herramientas que ayuden a las personas a verificar lo que están leyendo (Andersdotter, 2017). Hasta el momento las dos principales vías de actuación son la inclusión de programas de alfabetización en información orientados desde una perspectiva más cívica y la curación de contenidos, realizando guías temáticas que sean un punto de información sobre el fenómeno (Caridad-Sebastián et al., 2018).

Sin embargo, como argumenta Evelio Martínez las bibliotecas son un reflejo de la diversidad de ideas de la sociedad, por lo que un fondo documental puede y debe tener contenidos que sean falsos, inexactos 0 erróneos. Es decir, las bibliotecas no deben censurar y los bibliotecarios deben ser neutrales respecto a las fuentes que seleccionar (Martinez, 2018). Lo importante es dotar a los usuarios con las competencias informacionales para que desarrollen un criterio en el consumo informativo.

En este sentido, uno de los elementos más repetidos en el análisis bibliométrico realizado a partir de la búsqueda en Scopus es la alusión a la popular iniciativa de la IFLA y su sencilla infografía sobre cómo detectar noticias falsas. 
Figura 10: Infografía creada por la IFLA

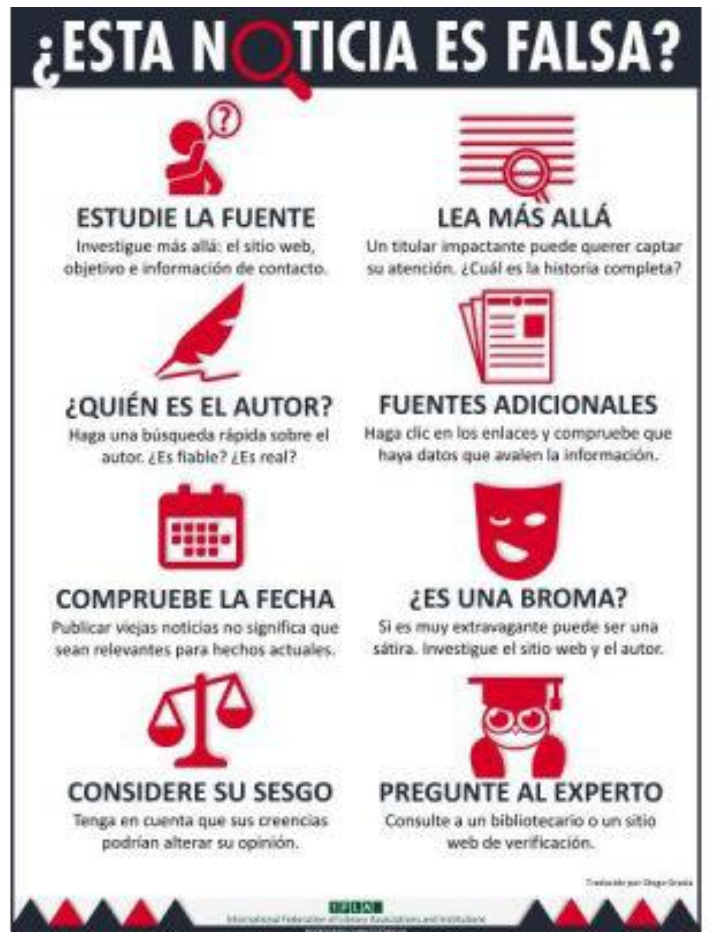

La biblioteca de la Harvard University proporciona información sobre antecedentes, enlaces y herramientas de organizaciones externas para ayudar a los usuarios a diferenciar noticias falsas. 
Figura11: Infografía de la biblioteca de la Harvard University que describe cinco formas de detectar y detener las noticias falsas

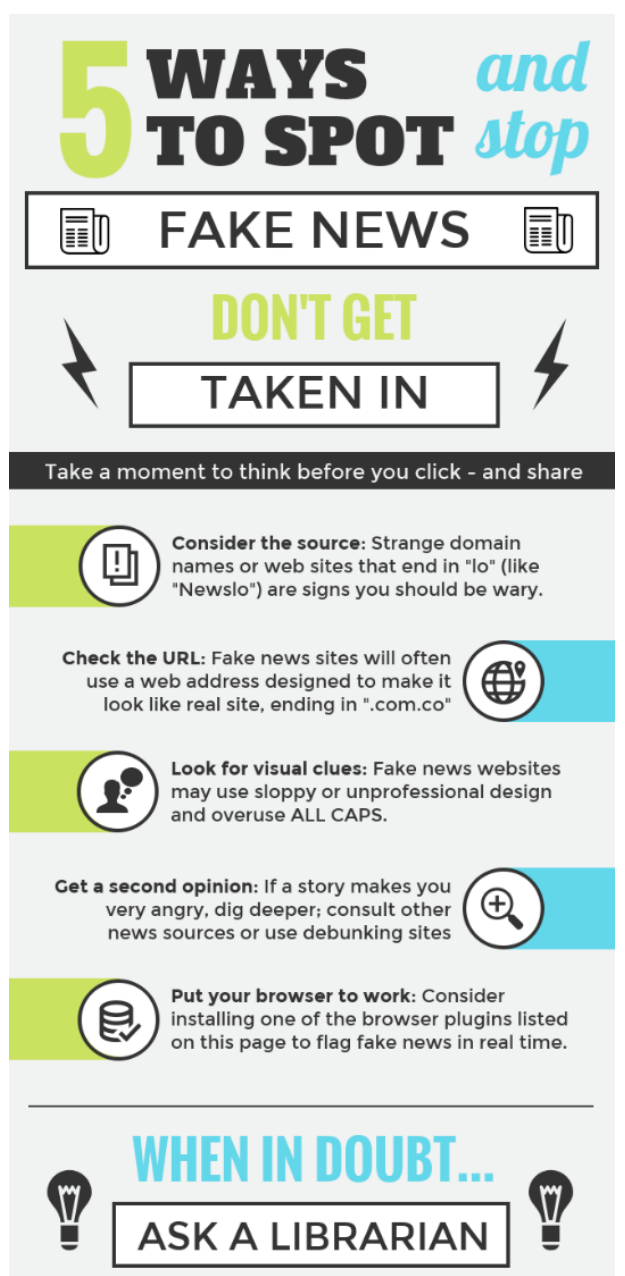

La biblioteca de la Indiana East University ofrece también en su web (http://iue.libguides.com/fakenews) una infografía propia, tutoriales e información práctica sobre las noticias falsas, distinguiendo cuatro categorías diferentes:

1. Sitios web falsos que se comparten a través de Facebook y otras redes sociales.

2. Sitios web por los que circula información engañosa o potencialmente no confiable.

3. Sitios web que en ocasiones usan clickbait

4. Sitios cómicos o de sátira que ofrecen importantes comentarios críticos sobre política y sociedad pero tienen el potencial de ser compartidos como noticias reales

A partir del material elaborado por la biblioteca de la Indiana East University unos bibliotecarios de Florida han desarrollado otra bajo el nombre de RADAR que detalla un plan que los estudiantes pueden seguir para 
examinar y evaluar las fuentes en los programas de alfabetización en información (Neely-Sardon \& Tignor, 2018).

Por último, destacar la creación de un juego de simulación para jóvenes sobre fake news desarrollado por científicos de la University of Cambridge y destinado para su uso en educación e investigación científica (Roozenbeek \& van der Linden, 2018).

Figura 12: Imagen tomada de https://www.getbadnews.com/

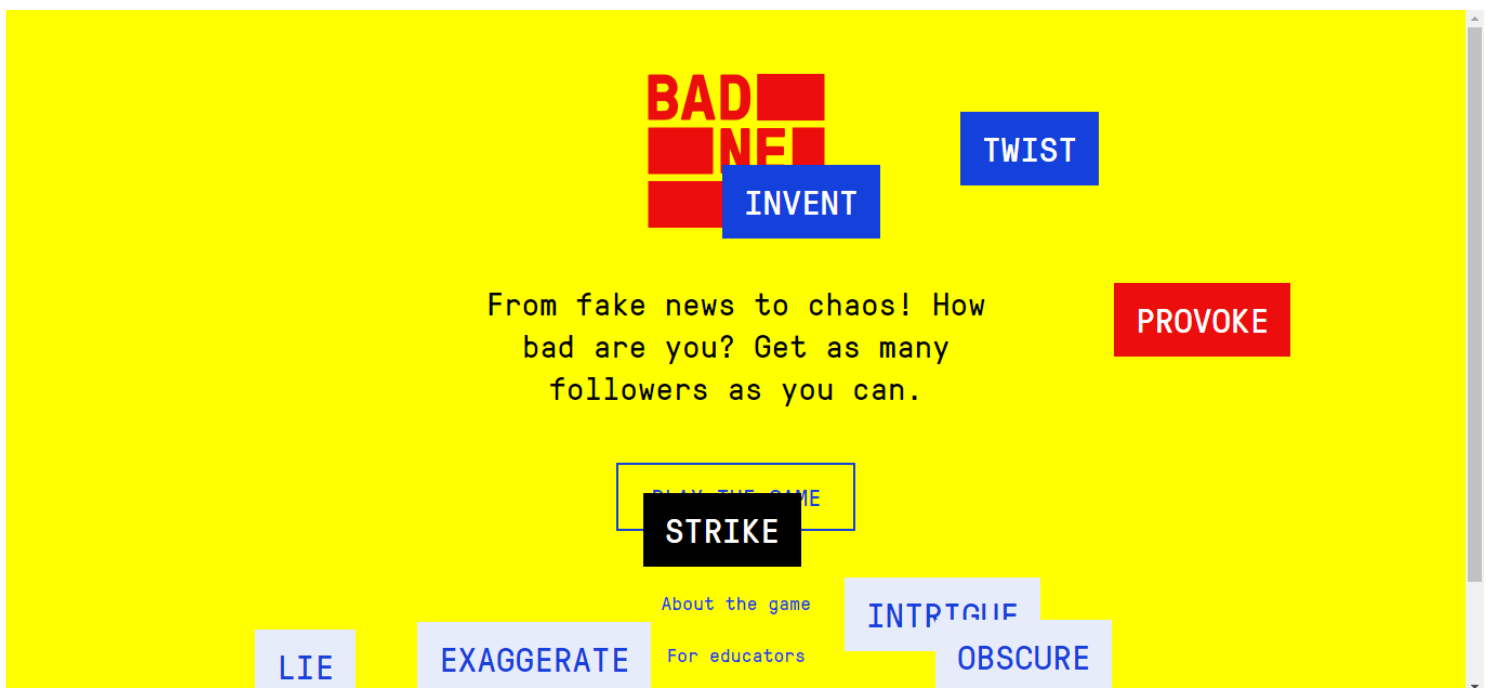

\section{Conclusiones}

El problema de la diseminación de la información falsa con la intención de engañar al público se ha convertido en un problema global, reforzado por la omnipresencia de Internet, la posibilidad de viralización y por el hecho que muchas personas reciben la mayoría de las noticias a través de las redes sociales y no saben reconocer la información falsa cuando se les presenta.

La lucha por diferenciar los hechos reales de los hechos de ficción forma parte de la condición humana y de la estrategia política. En cada época se han utilizado los medios existentes para difundir propaganda y falsedades, para informar o desinformar. Actualmente, la web ha cambiado la dinámica de la transmisión de la información y los hechos se mezclan con verdades y mentiras para generar mezclas informativas que impulsan y multiplican la especulación y el entretenimiento.

Además, la industria subversiva de fake news ha encontrado una buena oportunidad comercial de mercado. Es el caso de la empresa belga Media Vibes SNC que posee más de 180 sitios web dedicados a crear y difundir noticias falsas como, por ejemplo 24aktuelles.com. o react365.com. algunos de los cuales otorgan al usuario la posibilidad de generar sus propias fake news para difundirlas en sus redes sociales y engañar a todos sus amigos.

En nuestro estudio el $32 \%$ de los artículos analizados plantean algún tipo de solución para poder combatir las noticias falsas. La desinformación se puede contrarrestar de muchas maneras: cambiando los algoritmos de los motores de búsqueda o de los sitios de redes sociales, bloqueando ciertos sitios web, organizando 
campañas de concienciación o mediante la educación. El método más efectivo es fomentar consumidores de noticias críticos y bien informados. El conocimiento y la educación son, con mucho, las mejores armas contra las fake news y, en este contexto, y hasta que surja una solución mejor, la apuesta por la alfabetización mediática y el papel que pueden y deben ejercer los bibliotecarios es vital.

\section{Referencias bibliográficas}

Achter, P. (2008). Comedy in unfunny times: News parody and carnival after 9/11. Critical Studies in Media Communication, 25(3), 274-303. http://doi.org/10.1080/15295030802192038

Allcott, H., \& Gentzkow, M. (2017). Social media and fake news in the 2016 election. Journal of Economic Perspectives, 31(2), 211-236. http://doi.org/10.1257/jep.31.2.211

Andersdotter, K. (2017). Fatti alternativi e fake news: La verificabilità nella società dell'informazione. $A I B$ Studi, 57(1), 5-6. http://doi.org/10.2426/aibstudi-11618

Arif, N., Al-Jefri, M., Bizzi, I. H., Perano, G. B., Goldman, M., Haq, I., ... Ghezzi, P. (2018). Fake news or weak science? Visibility and characterization of antivaccine webpages returned by google in different languages and countries. Frontiers in Immunology, $g(J U N)$. http://doi.org/10.3389/fimmu.2018.01215

Arndt, S., \& Jones, D. S. (2018). Preventing sensationalistic science and fake news about substance use. Substance Abuse: Treatment, Prevention, and Policy, 13(1). http://doi.org/10.1186/s13011-0180148-3

Baider, F. H., \& Constantinou, M. (2017). Penelopegate: Fake news and online fake work over the 2017 French presidential campaign | Penelopegate: Fake news et retravail d'ethos en ligne lors de la campagne présidentielle de 2017. Studia Romanica Posnaniensia, 44(3), 9-27. http://doi.org/10.14746/strop.2017.443.001

Bardan, A. (2012). "The Tattlers" Tattle": Fake News, Linguistic National Intimacy, and New Media in Romania." Popular Communication, 19(1-2), 145-157. http://doi.org/10.1080/15405702.2012.638578

Baym, G. (2005). The Daily Show: Discursive integration and the reinvention of political journalism. Political Communication, 22(3), 259-276. http://doi.org/10.1080/10584600591006492

Berkowitz, D., \& Schwartz, D. A. (2016). Miley, CNN and the onion: When fake news becomes realer than real. Journalism Practice, 1Q(1), 1-17. http://doi.org/10.1080/17512786.2015.1006933

Berthon, P. R., \& Pitt, L. F. (2018). Brands, Truthiness and Post-Fact: Managing Brands in a Post-Rational World. Journal of Macromarketing, 38(2), 218-227. http://doi.org/10.1177/0276146718755869

Bharali, B., \& Goswami, A. L. (2018). Fake news: Credibility, cultivation syndrome and the new age media. Media Watch, 9(1), 118-130. http://doi.org/10.15655/mw/2018/v9i1/49277

Bhaskaran, H., Mishra, H., \& Nair, P. (2017). Contextualizing Fake News in Post-truth Era: Journalism Education in India. Asia Pacific Media Educator, 271), 41-50. http://doi.org/10.1177/1326365X17702277

Bolton, D. M., \& Yaxley, J. (2017). Fake news and clickbait - natural enemies of evidence-based medicine. BJU International, 119, 8-9. http://doi.org/10.1111/bju.13883 
Bozzola, E., Spina, G., Russo, R., Bozzola, M., Corsello, G., \& Villani, A. (2018). Mandatory vaccinations in European countries, undocumented information, false news and the impact on vaccination uptake: The position of the Italian pediatric society. Italian Journal of Pediatrics, 44(1). http://doi.org/10.1186/s13052-018-0504-y

Brandtzaeg, P. B., Følstad, A., \& Chaparro Domínguez, M. Á. (2017). How Journalists and Social Media Users Perceive Online Fact-Checking and Verification Services. Journalism Practice, 1-21. http://doi.org/10.1080/17512786.2017.1363657

Cardoso, G., Baldi, V., Pais, P. C., Paisana, M., Quintanilha, T. L., \& Couraceiro, P. (2018). As Fake News numa sociedade pós-verdade: Contextualização, potenciais soluções e análise. Lisboa: OberCom. http://doi.org/10.13140/RG.2.2.34603.75044

Chen, Y., Conroy, N. J., \& Rubin, V. L. (2015). Misleading online content: Recognizing clickbait as "false news." In WMDD 2015 - Proceedings of the ACM Workshop on Multimodal Deception Detection, co-located with ICMI 2015 (pp. 15-19). http://doi.org/10.1145/2823465.2823467

Chua, A. Y. K., \& Banerjee, S. (2018). Intentions to trust and share online health rumors: An experiment with medical professionals. Computers in Human Behavior, 87, 1-9. http://doi.org/10.1016/j.chb.2018.05.021

Cosentino, G. (2012). The Comical Inquisition: Striscia la Notizia and the Politics of Fake News on Italian Television. Popular Communication, 10(1-2), 52-65. http://doi.org/10.1080/15405702.2012.638570

Day, A., \& Thompson, E. (2012). Live From New York, It's the Fake News! Saturday Night Live and the (Non)Politics of Parody. Popular Communication, 10(1-2), 170-182. http://doi.org/10.1080/15405702.2012.638582

de los Santos, T. M., Smith, E., \& Cohen, M. (2018). Targeting Truth: How Museums Can Collaboratively Address Social Issues. Journal of Museum Education, 43(2), 104-113. http://doi.org/10.1080/10598650.2018.1457842

Del Vicario, M., Gaito, S., Quattrociocchi, W., Zignani, M., \& Zollo, F. (2018). News consumption during the Italian referendum: A cross-platform analysis on facebook and twitter. In Proceedings - 2017 International Conference on Data Science and Advanced Analytics, DSAA 2017 (Vol. 2018-Janua, pp. 648-657). http://doi.org/10.1109/DSAA.2017.33

Fischer, T. B. (2018). Editorial: IA, alternative facts and fake news-Is the post-factual turn starting to turn? Impact Assessment and Project Appraisal, 36(2), 129-130. http://doi.org/10.1080/14615517.2018.1426846

Harrington, S. (2012). From the "Little Aussie Bleeder" to Newstopia: (Really) Fake News in Australia. Popular Communication, 10(1-2), 27-39. http://doi.org/10.1080/15405702.2012.638571

Hauke, T. J., \& Herzig, V. (2017). Dangerous arachnids-Fake news or reality? Toxicon, 138, $173-183$. http://doi.org/10.1016/j.toxicon.2017.08.024

Hernández-González, V., Sans-Rosell, N., Jové-Deltell, M. C., \& Reverter-Masia, J. (2016). Comparación entre Web of Science y Scopus, Estudio Bibliométrico de las Revistas de Anatomía y Morfología. International Journal of Morphology. http://doi.org/10.4067/S0717-95022016000400032

Himma-Kadakas, M. (2017). Alternative facts and fake news entering journalistic content production cycle. Cosmopolitan Civil Societies, 9(2), 25-41. http://doi.org/10.5130/ccs.v9i2.5469 
Hofmann, B. (2018). Fake facts and alternative truths in medical research. BMC Medical Ethics, 19(1). http://doi.org/10.1186/s12910-018-0243-z

Ireland, S. (2018). Fake news alerts: teaching news literacy skills in a meme world. Reference Librarian, 17. http://doi.org/10.1080/02763877.2018.1463890

Johnson, B. G., \& Kelling, K. (2017). Placing Facebook: "Trending," "Napalm Girl," "fake news" and journalistic boundary work. Journalism Practice, 1-17. http://doi.org/10.1080/17512786.2017.1349546

Krishna, A. (2017). Motivation with misinformation: Conceptualizing lacuna individuals and publics as knowledge-deficient, issue-negative activists. Journal of Public Relations Research, 29(4), 176-193. http://doi.org/10.1080/1062726X.2017.1363047

Marcos Recio, J. C., Sánchez Vigil, J. M., \& Olivera Zaldua, M. (2017). La enorme mentira y la gran verdad de la información en tiempos de la postverdad. Scire.

McBrien, J., Dooley, K., \& Birman, D. (2017). Cultural and academic adjustment of refugee youth: Introduction to the special issue. International Journal of Intercultural Relations, 60, 104-108. http://doi.org/10.1016/j.ijintrel.2017.07.001

McNair, B. (2017). Fake news: Falsehood, fabrication and fantasy in Journalism. Fake News: Falsehood, Fabrication and Fantasy in Journalism. http://doi.org/10.4324/9781315142036

Meikle, G. (2012). "Find Out Exactly What to Think-Next!": Chris Morris, Brass Eye, and Journalistic Authority. Popular Communication, 10(1-2), 14-26. http://doi.org/10.1080/15405702.2012.638569

Neely-Sardon, A., \& Tignor, M. (2018). Focus on the facts: A news and information literacy instructional program. Reference Librarian, 1-14. http://doi.org/10.1080/02763877.2018.1468849

Pantumsinchai, P. (2018). Armchair detectives and the social construction of falsehoods: an actor-network approach. Information Communication and Society, 21(5), 761-778. http://doi.org/10.1080/1369118X.2018.1428654

Pauner Chulvi, C. (2018). Fake news and freedom of expression and information. The control of information contents on the network. TEORIA Y REALIDAD CONSTITUCIONAL, 41, 297-318.

Polage, D. C. (2012). Making up history: False memories of fake news stories. Europe's Journal of Psychology, 8(2), 245-250. http://doi.org/10.5964/ejop.v8i2.456

Roozenbeek, J., \& van der Linden, S. (2018). The fake news game: actively inoculating against the risk of misinformation. Journal of Risk Research, 1-11. http://doi.org/10.1080/13669877.2018.1443491

Shao, C., Hui, P.-M., Wang, L., Jiang, X., Flammini, A., Menczer, F., \& Ciampaglia, G. L. (2018). Anatomy of an online misinformation network. PLOS ONE, 13(4). http://doi.org/10.1371/journal.pone.0196087

Sommariva, S., Vamos, C., Mantzarlis, A., Đào, L. U., \& Martinez Tyson, D. (2018). Spreading the (Fake) News: Exploring Health Messages on Social Media and the Implications for Health Professionals Using a Case Study. American Journal of Health Education, 1-10. http://doi.org/10.1080/19325037.2018.1473178

Spohr, D. (2017). Fake news and ideological polarization: Filter bubbles and selective exposure on social media. Business Information Review, 34(3), 150-160. http://doi.org/10.1177/0266382117722446

Verma, N., Fleischmann, K. R., \& Koltai, K. S. (2017). Human values and trust in scientific journals, the mainstream media and fake news. Proceedings of the Association for Information Science and Technology, 54(1), 426-435. http://doi.org/10.1002/pra2.2017.14505401046 
Verrall, N., \& Mason, D. (2018). The taming of the shrewd: How can the military tackle sophistry, 'fake' news and post-truth in the digital age? RUSI Journal, 163(1), 20-28. http://doi.org/10.1080/03071847.2018.1445169

Vosoughi, S., Mohsenvand, M., \& Roy, D. (2017). Rumor gauge: Predicting the veracity of rumors on twitter. ACM Transactions on Knowledge Discovery from Data, 11(4). http://doi.org/10.1145/3070644

Waldrop, M. M. (2017). The genuine problem of fake news. Proceedings of the National Academy of Sciences of the United States of America, 114(48), 12631-12634. http://doi.org/10.1073/pnas.1719005114

Krishna, A. (2017). Motivation with misinformation: Conceptualizing lacuna individuals and publics as knowledge-deficient, issue-negative activists. Journal of Public Relations Research, 29(4), 176-193. https://doi.org/10.1080/1062726X.2017.1363047Achter, P. (2008). Comedy in unfunny times: News parody and carnival after 9/11. Critical Studies in Media Communication, 25(3), 274-303. http://doi.org/10.1080/15295030802192038

Allcott, H., \& Gentzkow, M. (2017). Social media and fake news in the 2016 election. Journal of Economic Perspectives, 31(2), 211-236. http://doi.org/10.1257/jep.31.2.211

Andersdotter, K. (2017). Fatti alternativi e fake news: La verificabilità nella società dell'informazione. $A I B$ Studi, 571), 5-6. http://doi.org/10.2426/aibstudi-11618

Arif, N., Al-Jefri, M., Bizzi, I. H., Perano, G. B., Goldman, M., Haq, I., ... Ghezzi, P. (2018). Fake news or weak science? Visibility and characterization of antivaccine webpages returned by google in different languages and countries. Frontiers in Immunology, $g(J U N)$. http://doi.org/10.3389/fimmu.2018.01215

Arndt, S., \& Jones, D. S. (2018). Preventing sensationalistic science and fake news about substance use. Substance Abuse: Treatment, Prevention, and Policy, 13(1). http://doi.org/10.1186/s13011-0180148-3

Baider, F. H., \& Constantinou, M. (2017). Penelopegate: Fake news and online fake work over the 2017 French presidential campaign | Penelopegate: Fake news et retravail d'ethos en ligne lors de la campagne présidentielle de 2017. Studia Romanica Posnaniensia, 44(3), 9-27. http://doi.org/10.14746/strop.2017.443.001

Bardan, A. (2012). "The Tattlers" Tattle": Fake News, Linguistic National Intimacy, and New Media in Romania." Popular Communication, 10(1-2), 145-157. http://doi.org/10.1080/15405702.2012.638578

Baym, G. (2005). The Daily Show: Discursive integration and the reinvention of political journalism. Political Communication, 22(3), 259-276. http://doi.org/10.1080/10584600591006492

Berkowitz, D., \& Schwartz, D. A. (2016). Miley, CNN and the onion: When fake news becomes realer than real. Journalism Practice, 1Q(1), 1-17. http://doi.org/10.1080/17512786.2015.1006933

Berthon, P. R., \& Pitt, L. F. (2018). Brands, Truthiness and Post-Fact: Managing Brands in a Post-Rational World. Journal of Macromarketing, 38(2), 218-227. http://doi.org/10.1177/0276146718755869

Bharali, B., \& Goswami, A. L. (2018). Fake news: Credibility, cultivation syndrome and the new age media. Media Watch, $9(1), 118-130$. http://doi.org/10.15655/mw/2018/v9i1/49277

Bhaskaran, H., Mishra, H., \& Nair, P. (2017). Contextualizing Fake News in Post-truth Era: Journalism Education in India. Asia Pacific Media Educator, 271), 41-50. http://doi.org/10.1177/1326365X17702277 
Bolton, D. M., \& Yaxley, J. (2017). Fake news and clickbait - natural enemies of evidence-based medicine. BJU International, 119, 8-9. http://doi.org/10.1111/bju.13883

Bozzola, E., Spina, G., Russo, R., Bozzola, M., Corsello, G., \& Villani, A. (2018). Mandatory vaccinations in European countries, undocumented information, false news and the impact on vaccination uptake: The position of the Italian pediatric society. Italian Journal of Pediatrics, 44(1). http://doi.org/10.1186/s13052-018-0504-y

Brandtzaeg, P. B., Følstad, A., \& Chaparro Domínguez, M. Á. (2017). How Journalists and Social Media Users Perceive Online Fact-Checking and Verification Services. Journalism Practice, 1-21. http://doi.org/10.1080/17512786.2017.1363657

Cardoso, G., Baldi, V., Pais, P. C., Paisana, M., Quintanilha, T. L., \& Couraceiro, P. (2018). As Fake News numa sociedade pós-verdade: Contextualização, potenciais soluções e análise. Lisboa: OberCom. http://doi.org/10.13140/RG.2.2.34603.75044

Chen, Y., Conroy, N. J., \& Rubin, V. L. (2015). Misleading online content: Recognizing clickbait as "false news." In WMDD 2015 - Proceedings of the ACM Workshop on Multimodal Deception Detection, co-located with ICMI 2015 (pp. 15-19). http://doi.org/10.1145/2823465.2823467

Chua, A. Y. K., \& Banerjee, S. (2018). Intentions to trust and share online health rumors: An experiment with medical professionals. Computers in Human Behavior, 87, 1-9. http://doi.org/10.1016/j.chb.2018.05.021

Cosentino, G. (2012). The Comical Inquisition: Striscia la Notizia and the Politics of Fake News on Italian Television. Popular Communication, 10(1-2), 52-65. http://doi.org/10.1080/15405702.2012.638570

Day, A., \& Thompson, E. (2012). Live From New York, It's the Fake News! Saturday Night Live and the (Non)Politics of Parody. Popular Communication, 10(1-2), 170-182. http://doi.org/10.1080/15405702.2012.638582

de los Santos, T. M., Smith, E., \& Cohen, M. (2018). Targeting Truth: How Museums Can Collaboratively Address Social Issues. Journal of Museum Education, 43(2), 104-113. http://doi.org/10.1080/10598650.2018.1457842

Del Vicario, M., Gaito, S., Quattrociocchi, W., Zignani, M., \& Zollo, F. (2018). News consumption during the Italian referendum: A cross-platform analysis on facebook and twitter. In Proceedings - 2017 International Conference on Data Science and Advanced Analytics, DSAA 2017 (Vol. 2018-Janua, pp. 648-657). http://doi.org/10.1109/DSAA.2017.33

Fischer, T. B. (2018). Editorial: IA, alternative facts and fake news-Is the post-factual turn starting to turn? Impact Assessment and Project Appraisal, 36(2), 129-130. http://doi.org/10.1080/14615517.2018.1426846

Harrington, S. (2012). From the "Little Aussie Bleeder" to Newstopia: (Really) Fake News in Australia. Popular Communication, 10(1-2), 27-39. http://doi.org/10.1080/15405702.2012.638571

Hauke, T. J., \& Herzig, V. (2017). Dangerous arachnids-Fake news or reality? Toxicon, 138, $173-183$. http://doi.org/10.1016/j.toxicon.2017.08.024

Hernández-González, V., Sans-Rosell, N., Jové-Deltell, M. C., \& Reverter-Masia, J. (2016). Comparación entre Web of Science y Scopus, Estudio Bibliométrico de las Revistas de Anatomía y Morfología. International Journal of Morphology. http://doi.org/10.4067/S0717-95022016000400032 
Himma-Kadakas, M. (2017). Alternative facts and fake news entering journalistic content production cycle. Cosmopolitan Civil Societies, 9(2), 25-41. http://doi.org/10.5130/ccs.v9i2.5469

Hofmann, B. (2018). Fake facts and alternative truths in medical research. BMC Medical Ethics, 19(1). http://doi.org/10.1186/s12910-018-0243-z

Ireland, S. (2018). Fake news alerts: teaching news literacy skills in a meme world. Reference Librarian, 17. http://doi.org/10.1080/02763877.2018.1463890

Johnson, B. G., \& Kelling, K. (2017). Placing Facebook: "Trending," "Napalm Girl," "fake news" and journalistic boundary work. Journalism Practice, 1-17. http://doi.org/10.1080/17512786.2017.1349546

Krishna, A. (2017). Motivation with misinformation: Conceptualizing lacuna individuals and publics as knowledge-deficient, issue-negative activists. Journal of Public Relations Research, 29(4), 176-193. http://doi.org/10.1080/1062726X.2017.1363047

Marcos Recio, J. C., Sánchez Vigil, J. M., \& Olivera Zaldua, M. (2017). La enorme mentira y la gran verdad de la información en tiempos de la postverdad. Scire.

McBrien, J., Dooley, K., \& Birman, D. (2017). Cultural and academic adjustment of refugee youth: Introduction to the special issue. International Journal of Intercultural Relations, 60, 104-108. http://doi.org/10.1016/j.ijintrel.2017.07.001

McNair, B. (2017). Fake news: Falsehood, fabrication and fantasy in Journalism. Fake News: Falsehood, Fabrication and Fantasy in Journalism. http://doi.org/10.4324/9781315142036

Meikle, G. (2012). "Find Out Exactly What to Think-Next!": Chris Morris, Brass Eye, and Journalistic Authority. Popular Communication, 10(1-2), 14-26. http://doi.org/10.1080/15405702.2012.638569

Neely-Sardon, A., \& Tignor, M. (2018). Focus on the facts: A news and information literacy instructional program. Reference Librarian, 1-14. http://doi.org/10.1080/02763877.2018.1468849

Pantumsinchai, P. (2018). Armchair detectives and the social construction of falsehoods: an actor-network approach. Information Communication and Society, 21(5), 761-778. http://doi.org/10.1080/1369118X.2018.1428654

Pauner Chulvi, C. (2018). Fake news and freedom of expression and information. The control of information contents on the network. TEORIA Y REALIDAD CONSTITUCIONAL, 41, 297-318.

Polage, D. C. (2012). Making up history: False memories of fake news stories. Europe's Journal of Psychology, 8(2), 245-250. http://doi.org/10.5964/ejop.v8i2.456

Roozenbeek, J., \& van der Linden, S. (2018). The fake news game: actively inoculating against the risk of misinformation. Journal of Risk Research, 1-11. http://doi.org/10.1080/13669877.2018.1443491

Shao, C., Hui, P.-M., Wang, L., Jiang, X., Flammini, A., Menczer, F., \& Ciampaglia, G. L. (2018). Anatomy of an online misinformation network. PLOS ONE, 13(4). http://doi.org/10.1371/journal.pone.0196087

Sommariva, S., Vamos, C., Mantzarlis, A., Đào, L. U., \& Martinez Tyson, D. (2018). Spreading the (Fake) News: Exploring Health Messages on Social Media and the Implications for Health Professionals Using a Case Study. American Journal of Health Education, 1-10. http://doi.org/10.1080/19325037.2018.1473178

Spohr, D. (2017). Fake news and ideological polarization: Filter bubbles and selective exposure on social media. Business Information Review, 34(3), 150-160. http://doi.org/10.1177/0266382117722446

Verma, N., Fleischmann, K. R., \& Koltai, K. S. (2017). Human values and trust in scientific journals, the 
mainstream media and fake news. Proceedings of the Association for Information Science and Technology, 54(1), 426-435. http://doi.org/10.1002/pra2.2017.14505401046

Verrall, N., \& Mason, D. (2018). The taming of the shrewd: How can the military tackle sophistry, 'fake' news and post-truth in the digital age? RUSI Journal, 163(1), 20-28. http://doi.org/10.1080/03071847.2018.1445169

Vosoughi, S., Mohsenvand, M., \& Roy, D. (2017). Rumor gauge: Predicting the veracity of rumors on twitter. ACM Transactions on Knowledge Discovery from Data, 11(4). http://doi.org/10.1145/3070644

Waldrop, M. M. (2017). The genuine problem of fake news. Proceedings of the National Academy of Sciences of the United States of America, 114(48), 12631-12634. http://doi.org/10.1073/pnas.1719005114 